Research Paper

\title{
The Minnesota mobile extracorporeal cardiopulmonary resuscitation consortium for treatment of out-of-hospital refractory ventricular fibrillation: Program description, performance, and outcomes
}

\author{
Jason A. Bartos ${ }^{\mathrm{a}, \mathrm{b}}$, R.J. Frascone ${ }^{\mathrm{b}, \mathrm{c}}$, Marc Conterato ${ }^{\mathrm{b}, \mathrm{d}}$, Keith Wesley ${ }^{\mathrm{e}}$, Charles Lick, \\ Kevin Sipprellg, ${ }^{\text {, Nik Vuljaj }}{ }^{\mathrm{e}}$, Aaron Burnett ${ }^{\mathrm{h}}$, Bjorn K Peterson ${ }^{\mathrm{i}}$, Nicholas Simpson ${ }^{\mathrm{j}}$, Kealy Ham ${ }^{\mathrm{k}}$, \\ Charles Bruen $^{\mathrm{k}}$, Casey Woster ${ }^{\mathrm{k}}$, Kari B Haley ${ }^{\mathrm{k}}$, Joanna Moore ${ }^{\mathrm{j}}$, Brandon Trigger ${ }^{\mathrm{l}}$, \\ Lucinda Hodgson $^{\mathrm{b}}$, Kim Harkins ${ }^{\mathrm{b}}$, Marinos Kosmopoulos ${ }^{\mathrm{b}}$, Tom P. Aufderheide ${ }^{\mathrm{m}}$, Jakub Tolar ${ }^{\mathrm{n}}$, \\ Demetris Yannopoulos ${ }^{\mathrm{a}, \mathrm{b}, *}$
}

\footnotetext{
a Division of Cardiology, Department of Medicine, University of Minnesota School of Medicine, Minneapolis, MN, United States

${ }^{\mathrm{b}}$ Center for Resuscitation Medicine, University of Minnesota School of Medicine, Minneapolis, MN 55401, United States

${ }^{\mathrm{c}}$ Department of Emergency Medicine, Regions Hospital, St. Paul, MN, United States

${ }^{\mathrm{d}}$ Department of Emergency Medicine, and North Memorial EMS, North Memorial Medical Center, Robbinsdale, MN, United States

${ }^{\mathrm{e}} M$ Health Fairview Emergency Medical Services, MN, United States

${ }^{\mathrm{f}}$ Allina Health Emergency Medicinal Services, MN, United States

${ }^{g}$ Ridgeview Emergency Medicinal Services, MN, United States

${ }^{\mathrm{h}}$ Woodbury and Cottage Grove, Emergency Medical Services, MN, United States

i Lakeview and Maplewood Emergency Medical Services, United States

${ }^{\mathrm{j}}$ Hennepin County Emergency Medical Services, Hennepin County, Minneapolis Minnesota, United States

${ }^{\mathrm{k}}$ Regions Hospital, Health Partners, St Paul Minnesota, United States

${ }^{1}$ M Heath Fairview Southdale Hospital Emergency Medicine Department, United States

${ }^{\mathrm{m}}$ Department of Emergency Medicine, Medical College of Wisconsin, Milwaukee WI, United States

${ }^{\mathrm{n}}$ Dean of the Medical School and Vice President of Clinical affairs at the University of Minnesota, Minneapolis, MN, United States
}

\section{A R T I C L E I N F O}

\section{Article History:}

Received 25 October 2020

Revised 30 October 2020

Accepted 3 November 2020

Available online 13 November 2020

\section{Keywords:}

Cardiac arrest

Extracorporeal cardiopulmonary resuscitation

Extracorporeal membrane oxygenation

Sudden cardiac death

Refractory ventricular fibrillation

\begin{abstract}
A B S T R A C T
Background: We describe implementation, evaluate performance, and report outcomes from the first program serving an entire metropolitan area designed to rapidly deliver extracorporeal membrane oxygenation (ECMO)-facilitated resuscitation to patients with refractory ventricular fibrillation/ventricular tachycardia (VF/VT) out-of-hospital cardiac arrest (OHCA).

Methods: This observational cohort study analyzed consecutive patients prospectively enrolled in the Minnesota Mobile Resuscitation Consortium's ECMO-facilitated resuscitation program. Entry criteria included: 1) adults (aged 18-75), 2) VF/VT OHCA, 3) no return of spontaneous circulation following 3 shocks, 4) automated cardiopulmonary resuscitation with a Lund University Cardiac Arrest System (LUCAS ${ }^{\mathrm{TM}}$ ), and 5) estimated transfer time of $<30 \mathrm{~min}$. The primary endpoint was functionally favorable survival to hospital discharge with Cerebral Performance Category (CPC) 1 or 2. Secondary endpoints included 3-month functionally favorable survival, program benchmarks, ECMO cannulation rate, and safety. Essential program components included emergency medical services, 3 community ECMO Initiation Hospitals with emergency department ECMO cannulation sites and 24/7 cardiac catheterization laboratories, a 24/7 mobile ECMO cannulation team, and a single, centralized ECMO intensive care unit.

Findings: From December 1, 2019 to April 1, 2020, 63 consecutive patients were transported and 58 (97\%) met criteria and were treated by the mobile ECMO service. Mean age was $57 \pm 1.8$ years; $46 / 58$ (79\%) were male. Program benchmarks were variably met, $100 \%$ of patients were successfully cannulated, and no safety issues were identified. Of the 58 patients, 25/58 (43\% [CI:31-56\%]) were both discharged from the hospital and alive at 3 months with CPC 1 or 2.
\end{abstract}

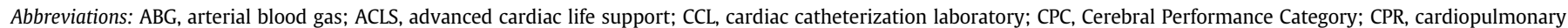

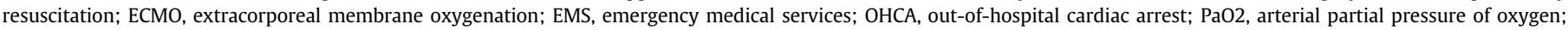
ROSC, return of spontaneous circulation; SEM, standard error of the mean; VF/VT, ventricular fibrillation/ventricular tachycardia

* Corresponding author at: Center for Resuscitation Medicine, University of Minnesota School of Medicine, Minneapolis, MN 55401, United States.

E-mail address: yanno001@umn.edu (D. Yannopoulos). 
Interpretation: This first, community-wide ECMO-facilitated resuscitation program in the US demonstrated $100 \%$ successful cannulation, $43 \%$ functionally favorable survival rates at hospital discharge and 3 months, as well as safety. The program provides a potential model of this approach for other communities.

Funding: The Helmsley Charitable Trust

(c) 2020 The Authors. Published by Elsevier Ltd. This is an open access article under the CC BY-NC-ND license (http://creativecommons.org/licenses/by-nc-nd/4.0/)

\section{Research in Context}

\section{Evidence before this study}

Multiple observational cohort studies have demonstrated the potential benefit of extracorporeal membrane oxygenation (ECMO) for treatment of patients with refractory cardiac arrest. However, the time from arrest to ECMO initiation is a key predictor of survival with a $25 \%$ increase in mortality for every $10 \mathrm{~min}$ of CPR beyond $30 \mathrm{~min}$.

\section{Added value of this study}

This study demonstrates that ECMO can be delivered across a metropolitan area safely with a specialized team, meeting patients at predetermined ECMO cannulation sites closer to the location of the patient's arrest. The rate of neurologically favorable survival (43\%) was similar to prior ECMO studies with geographically constrained cohorts as well as the newly published prospective, randomized, single center ARREST trial.

\section{Implications of all the available evidence}

ECMO can provide improved survival for patients with refractory VF/VT out-of-hospital cardiac arrest using a well-designed, comprehensive community-wide resuscitation program. Extensive training and collaboration are required across multiple EMS and healthcare systems to effectively identify patients, safely provide these resources, and achieve favorable survival rates to the target population.

\section{Introduction}

Out-of-hospital cardiac arrest (OHCA) affects approximately 360,000 patients/year in the United States with a survival rate of $<$ $10 \%$ [1-3]. Although OHCA patients presenting to emergency medical services (EMS) with a shockable rhythm, including ventricular fibrillation/ventricular tachycardia (VF/VT), have better outcomes (30\% functionally favorable survival rates overall), [3,4] 50\% of these patients are refractory to standard advanced cardiac life support (ACLS) treatment [4-6].

Extracorporeal membrane oxygenation (ECMO), followed by immediate coronary angiography and percutaneous coronary intervention (PCI), called ECMO-facilitated resuscitation, has been used to treat refractory VF/VT OHCA [7-12]. The survival to hospital discharge rate of this approach was studied in the prospective, randomized, single center ARREST Trial, demonstrating a rate of $42.9 \%$ with ECMO-facilitated resuscitation compared to $6.7 \%$ with standard ACLS treatment [13]. Whether this approach can be routinely provided outside this single center experience is unknown.

The purpose of this observational study is to describe implementation, evaluate program performance, and report outcomes from the first refractory VF/VT OHCA, ECMO-facilitated resuscitation program in the United States serving an entire metropolitan community. This reported experience was performed with complete independence and without patient overlap to the ARREST Trial [13]. Its unique program structure included using mobile ECMO cannulation teams, 3 strategically located ECMO Initiation Hospitals, each housing both an emergency department (ED) ECMO cannulation site and a 24/7 cardiac catheterization laboratory $(\mathrm{CCL})$, and a single, centralized ECMO intensive care unit (ICU). We hypothesized that the program would successfully provide 24/7 ECMO-facilitated resuscitation to the entire metropolitan community, demonstrate safety, and achieve functionally favorable survival rates consistent with our previously published case series from a single center [5,7-9].

\section{Methods}

The Center for Resuscitation at the University of Minnesota in Minneapolis established the Minnesota Resuscitation Consortium (MRC) in 2012 with the goal to advance research, education, and community engagement in the field of resuscitation. In 2015, a single center (the University of Minnesota) developed an approach for ECMO-facilitated resuscitation treatment of refractory VF/VT OHCA, publishing multiple case series of its survival potential [5,7-9]. By 2017, community consensus had been achieved to extend a new ECMO-facilitated resuscitation program to the entire metropolitan community.

\section{Program development}

Inclusion of all community stakeholders was fundamental to development of the new program, involving four healthcare systems, ten EMS systems, governmental officials, hospital administrative leadership, and key clinical leaders throughout the Minneapolis-St. Paul metropolitan area. Developmental meetings established fundamental components: 1) a single, centralized ECMO ICU at the University of Minnesota for delivery of post-arrest care (experienced caring for this challenging patient population with 24/7 surgical and medical specialist availability), 2) 3 additional, ECMO Initiation Hospitals at strategic geographic locations to extend the ECMO-facilitated resuscitation footprint, with ED ECMO Cannulation Site capability, and 24/7 CCL availability at each ECMO Initiation Hospital, 3) a core, 24/7 mobile ECMO cannulation team, 4) four dedicated ECMO cannulation team rapid response vehicles, 5) standard operating procedures, hospital privileges, certifications, training programs, and contracts for services, 6) community-wide data collection and performance benchmarks, and 7) maximal likelihood of economic and organizational sustainability. Development began in February 2017 and the community-wide program was activated on December 1, 2019.

\section{System organization}

The Minnesota Mobile Resuscitation Consortium (MMRC) was established as a nonprofit entity. Representatives from each healthcare system had positions on the MMRC board of directors providing direct input and future planning. The MMRC coordinated training EMS providers, hiring and training mobile ECMO cannulation team members from each healthcare system, training and credentialing team members at each ECMO Initiation Hospital, supply acquisition and resupply at each ECMO Initiation Hospital ED Cannulation Site, documentation and billing, cost sharing across healthcare systems, and performance evaluation. All services were provided 24/7 at every ECMO Initiation Hospital and the single, centralized ECMO ICU. 
A

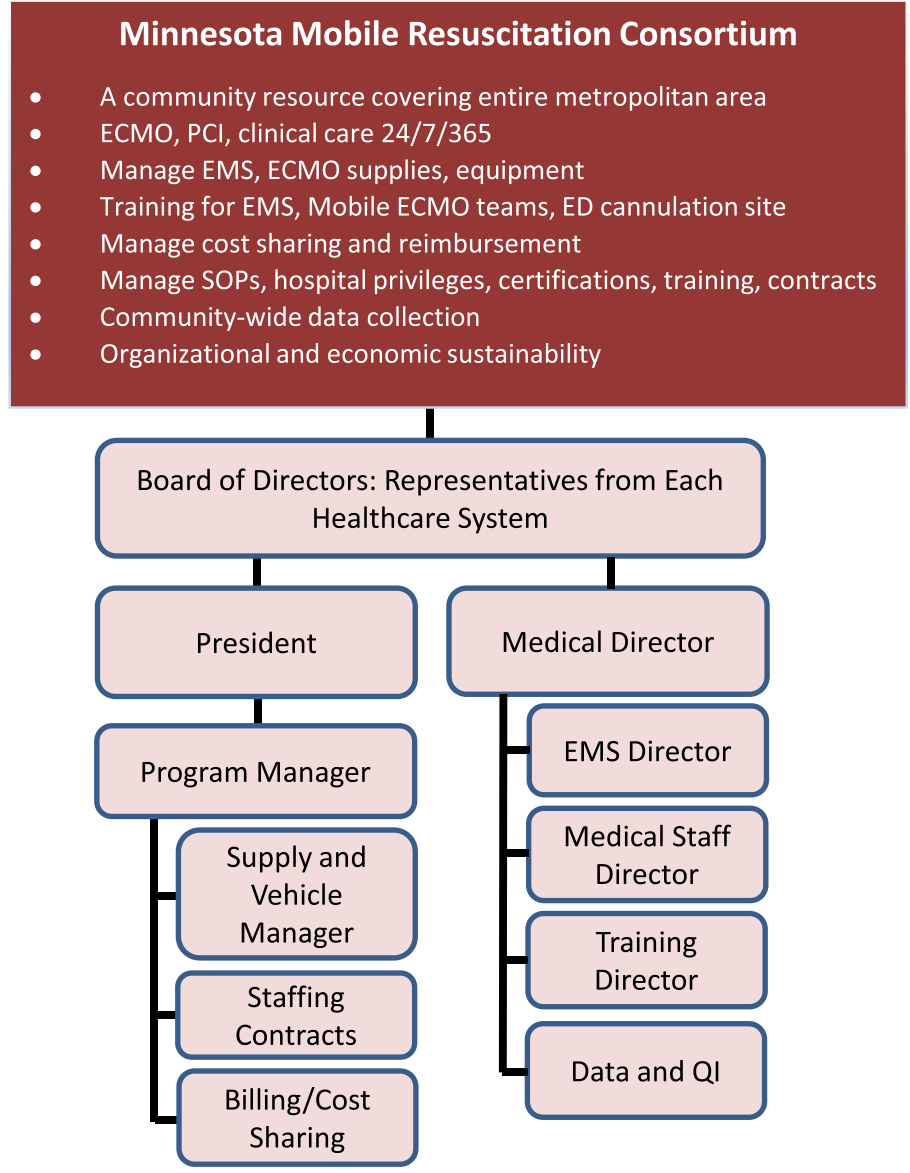

B

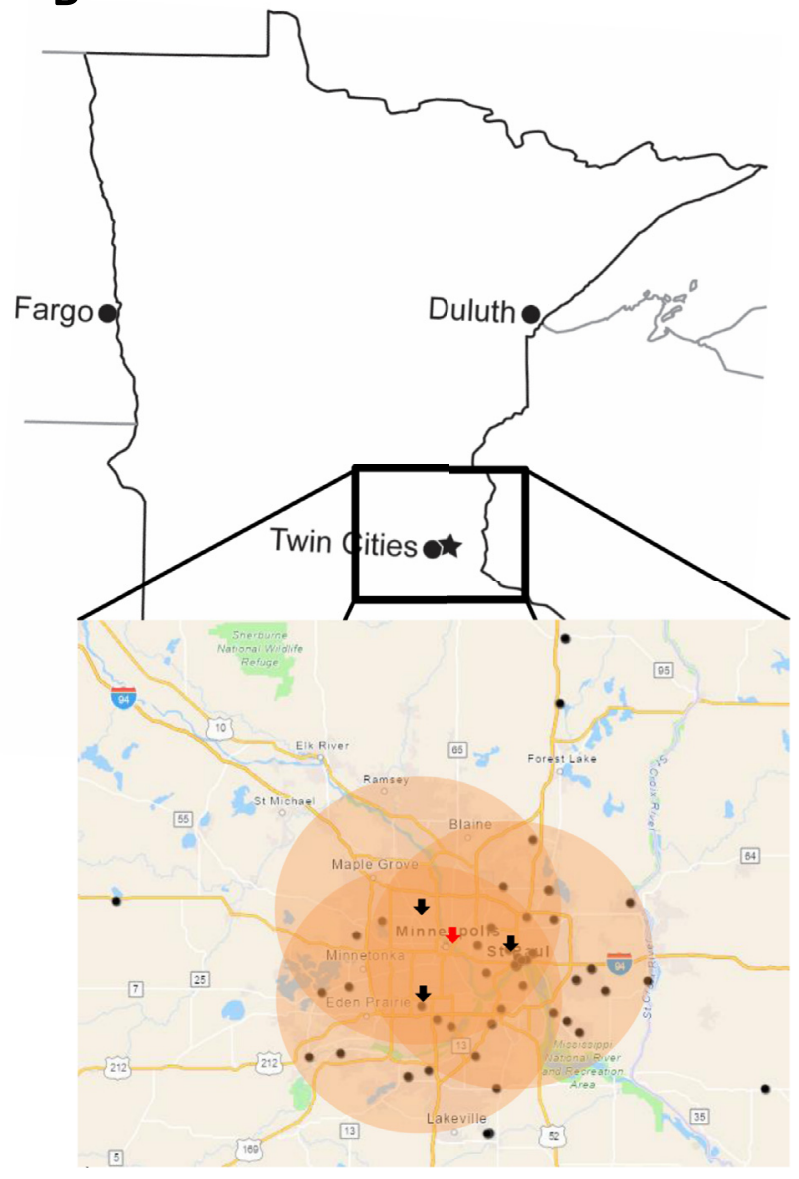

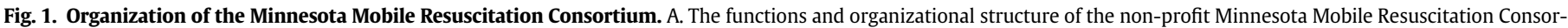

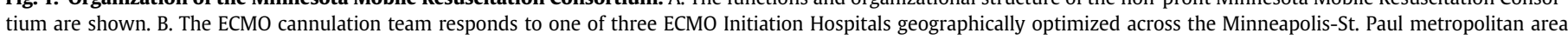

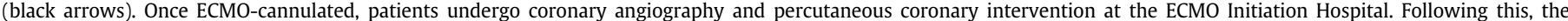

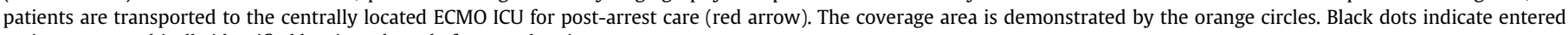
patients geographically identified by zip code and often overlapping.

MMRC organization and geographic coverage is shown in Fig. $1 \mathrm{~A}$ and $\mathrm{B}$.

\section{Mobile ECMO cannulation team}

Each mobile ECMO cannulation team consisted of three members: one senior cannulating physician, one sterile assistant, and one non-sterile assistant. Assistants were critical care-experienced paramedics/nurses or physicians. Ten physicians and 9 critical careexperienced paramedics/nurses from all four healthcare systems participated. Physicians performing the cannulations were specialized in interventional cardiology, emergency medicine, and critical care. Team members were on-call for 12 or 24-hour shifts with benchmark activation to ED ECMO Cannulation site time of < 15 min. Team members completed emergency vehicle operations courses allowing them to drive dedicated rapid response vehicles. The team carried all necessary cannulation supplies to the ED cannulation site, although some supplies were kept on-site to speed preparation. The team stayed at the patient's bedside from ED cannulation through transport to the single, centralized ECMO ICU, providing ongoing resuscitation and ECMO management, as needed. The process for team activation, patient stabilization, and transport is shown in Fig. 2.

\section{Training}

Mobile ECMO cannulation team members were recruited from participating healthcare systems. Given varied medical backgrounds, extensive training ensured achievement of similar skillsets. Training included $16 \mathrm{~h}$ didactic lecture/discussion and $12 \mathrm{~h}$ cannulation simulation and trouble-shooting. Members were trained in all aspects of the equipment including supplies needed for ECMO cannulation, ECMO initiation, equipment troubleshooting, resuscitation techniques, and treatments to optimize the first hours of acute care and stabilization. Successful performance of the cannulation procedure and demonstration of troubleshooting proficiency was required in simulation prior to progression to clinical training. Upon completion, each physician was credentialed by the healthcare systems to begin as a trainee. Clinical training included observation, the assistant role, followed by the primary cannulator role under supervision. Clinical proficiency was assessed by successful completion of at least 5 cannulations in the primary cannulator role followed by appropriate resuscitation and troubleshooting of alarms and post-arrest pathology. Consensus of the senior cannulators was required prior to advancement to independent cannulator. Once acceptable performance was confirmed, they were certified and credentialed for independent practice. All trainees successfully completed the training program. 


\section{Out-of-Hospital Care}

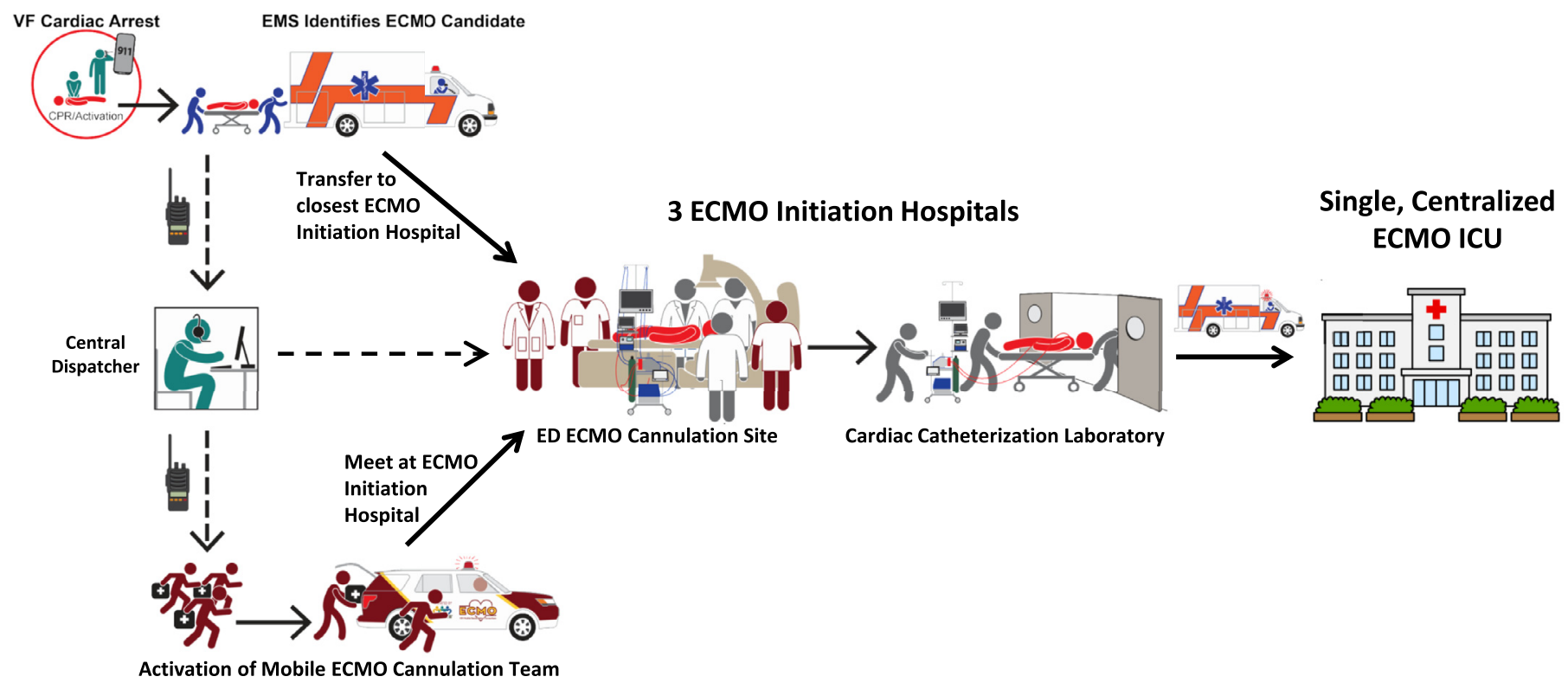

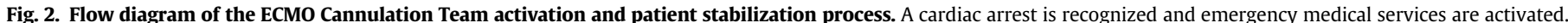

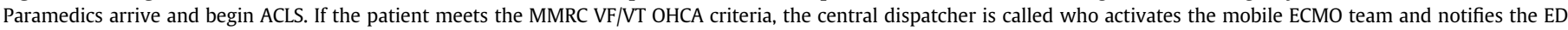

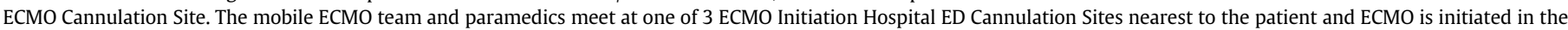

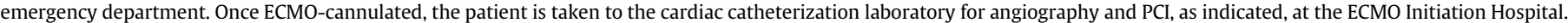
The patient is then transported to the single, centralized ECMO ICU for post-arrest care.

Ongoing quality improvement and skill maintenance was accomplished by participation in case review conferences, monitoring and feedback on management of ECMO complications (Extracorporeal Life Support Organization [ELSO] criteria [14]), ongoing discussions of advancements in resuscitation, and repeated simulation cannulation training. Quarterly skill assessment was performed to verify maintenance of skills.

EMS medical directors assisted training EMS providers. This practice change represented a paradigm shift from prolonged on-scene resuscitation to accurate identification of eligible refractory VF/VT patients, early dispatch notification, and rapid transport with ongoing ACLS. Feedback was provided in each case reinforcing minimal onscene time, airway management, appropriate ventilation, medical therapy, and ACLS care during transport.

\subsection{Study design}

This observational cohort study analyzes the first 63 consecutive patients in the MMRC's ECMO-facilitated resuscitation protocol from December 1, 2019 to April 1, 2020. The Institutional Review Board at the University of Minnesota approved this study (No. 1703M11301) with waiver of informed consent.

The primary study endpoint was functionally favorable survival to hospital discharge (Cerebral Performance Category [CPC] of 1 or 2) assessed in-person by the discharging physician. Secondary endpoints included 3-month survival, 3-month functionally favorable survival (assessed in-person by follow up physicians), program performance and benchmarks, successful cannulation rate, and safety (program safety and cannulation complications).

This report adheres to the STROBE guidelines for observational studies in epidemiology [15].

\subsection{MMRC ECMO-facilitated resuscitation protocol}

Patient selection criteria were previously published [5]. Briefly, patients meeting the following criteria were transported by EMS to the closest participating ECMO Initiation Hospital ED cannulation site: 1) adults (aged 18-75), 2) VF/VT OHCA, 3) no return of spontaneous circulation (ROSC) following 3 shocks, 4) automated cardiopulmonary resuscitation with a Lund University Cardiac Arrest System $\left(\right.$ LUCAS $\left.^{\mathrm{TM}}\right)$, and 5) estimated transfer time of $<30$ min. Exclusions were nursing home residents, known DNR orders, significant bleeding, or known terminal illness with less than one-year life expectancy. These inclusion criteria are identical to the ARREST Trial inclusion criteria [13].

On eligible patient identification, paramedics called a central dispatcher who then notified the mobile ECMO cannulation team and the ED ECMO cannulation site (Fig. 2). Paramedics transported with ongoing mechanical CPR and ACLS. The mobile ECMO cannulation team also traveled to the ECMO Initiation Hospital ED cannulation site and prepared equipment in a pre-specified room in the ED. On patient arrival, an arterial blood gas and lactic acid were assessed to determine resuscitation discontinuation criteria. Patients meeting $\geq 2$ resuscitation discontinuation criteria (previously established as metabolic status unlikely to benefit [5, 7-9]) were declared dead: 1 ) end-tidal $\mathrm{CO}_{2} \leq 10 \mathrm{mmHg}, 2$ ) arterial partial pressure of oxygen $\left(\mathrm{PaO}_{2}\right) \leq 50 \mathrm{mmHg}$ or $\mathrm{O}_{2}$ saturation $\leq 85 \%$, or 3) lactic acid $\geq 18 \mathrm{mmol} / \mathrm{L}$. Patients without these criteria underwent emergent ultrasound-guided percutaneous ECMO cannulation by the mobile ECMO cannulation team in the ED while ED physicians continued resuscitation. Dedicated ED fluoroscopy confirmed wire and cannula position. Patients achieving hemodynamically stable ROSC during transport were treated by ED staff per standard practice.

ECMO patients were stabilized with pressors, as needed, and the cannulas secured. The CCL was then paged and mobilized. Next, patients underwent computerized tomography with noncontrast head, chest, abdomen, and pelvis scans assessing CPRrelated trauma. Following computerized tomography, patients proceeded to the CCL for coronary angiography and $\mathrm{PCI}$, as needed. Patients were then transferred to the single, centralized ECMO ICU (Fig. 2). 
Table 1

MMRC program performance metrics and benchmarks.

\begin{tabular}{|c|c|}
\hline Performance Metric & Benchmark \\
\hline \multicolumn{2}{|l|}{10 EMS Agencies } \\
\hline \multicolumn{2}{|l|}{ Time from 911 to first responder arrival } \\
\hline EMS on-scene time & $<15 \min$ \\
\hline \multicolumn{2}{|l|}{ Time from 911 to patient arrival at ED } \\
\hline \multicolumn{2}{|l|}{ Accuracy of patient selection } \\
\hline \multicolumn{2}{|l|}{ ECMO Initiation Hospitals } \\
\hline \multicolumn{2}{|l|}{ Number/proportion of patients treated } \\
\hline \multicolumn{2}{|l|}{ Discontinuation Criteria on ED Arrival } \\
\hline \multicolumn{2}{|l|}{ Initial end-tidal $\mathrm{CO} 2$} \\
\hline \multicolumn{2}{|l|}{$\mathrm{PaO} 2$} \\
\hline \multicolumn{2}{|l|}{$\mathrm{O} 2$ saturation } \\
\hline \multicolumn{2}{|l|}{ Lactic acid } \\
\hline \multicolumn{2}{|l|}{$\begin{array}{l}\text { Proportion of patients meeting discontinuation } \\
\text { criteria on ED arrival }\end{array}$} \\
\hline \multicolumn{2}{|l|}{ Mobile ECMO cannulation team } \\
\hline Response time to ED & $<15 \min$ \\
\hline \multicolumn{2}{|l|}{ Number and safety of rapid responses } \\
\hline Patient ED arrival to ECMO cannulation time & $<15 \min$ \\
\hline Total duration of professional CPR & $<60 \min$ \\
\hline \multicolumn{2}{|l|}{ Cannulation success rate } \\
\hline \multicolumn{2}{|l|}{ Cannulation complication rate (ELSO Criteria) } \\
\hline \multicolumn{2}{|l|}{ Cardiac Catherization Laboratory (CCL) } \\
\hline Time from 911 to CCL arrival & $<120 \min$ \\
\hline \multicolumn{2}{|l|}{ Incidence of severe coronary artery disease } \\
\hline \multicolumn{2}{|l|}{ significant lesions as determined by the } \\
\hline \multicolumn{2}{|l|}{ interventional cardiologist) } \\
\hline \multicolumn{2}{|l|}{ Number/proportion of patients receiving PCI } \\
\hline \multicolumn{2}{|l|}{ Transport to the ECMO ICU } \\
\hline \multicolumn{2}{|l|}{ Adverse events } \\
\hline \multicolumn{2}{|l|}{ ECMO ICU } \\
\hline \multicolumn{2}{|l|}{ Ejection fraction on hospital discharge } \\
\hline \multicolumn{2}{|l|}{ Duration of hospitalization in survivors } \\
\hline \multicolumn{2}{|l|}{ Duration of hospitalization in non-survivors } \\
\hline \multicolumn{2}{|l|}{ ECMO-related complications (ELSO criteria) } \\
\hline Outcomes & \\
\hline Survival to hospital discharge & \\
\hline Survival to hospital discharge with CPC of $1-2$ & \\
\hline Survival to 3 months & \\
\hline Survival to 3 months with CPC of $1-2$ & \\
\hline
\end{tabular}

Table 1: MMRC Program Performance Metrics and Benchmarks. Values are $\mathrm{n}(\%)$ or mean \pm standard deviation. MMRC $=$ Minnesota Mobile Resuscitation Consortium EMS $=$ Emergency Medical Service, $\mathrm{ED}=$ Emergency Department; $\mathrm{ECMO}=$ Extracorporeal Membrane Oxygenation, $\mathrm{CO} 2$ = carbon dioxide; $\mathrm{PaO} 2=$ partial pressure of oxygen in arterial blood; $\mathrm{O} 2$ = oxygen; $\mathrm{CPR}=$ cardiopulmonary resuscitation; $\mathrm{CCL}=$ Cardiac Catheterization Laboratory, $\mathrm{PCI}=$ percutaneous coronary intervention; ICU = intensive care unit; ELSO = Extracorporeal Life Support Organization ${ }^{14}$; $\mathrm{CPC}=$ Cerebral Performance Category.

\subsection{Performance and benchmark monitoring}

Secure data acquisition was established to benchmark and monitor performance for each component of the program. Data was acquired and managed by MMRC, housed at the University of Minnesota, and routinely reported at board meetings. Performance metrics and benchmarks were centrally monitored and established a priori as shown in Table 1.

\section{Data management and statistical analysis}

Relevant data were recorded in a REDCap database and exported to excel format for biostatistical analysis. Data are presented as mean \pm standard deviation (SD) for continuous variables, and frequency (percentage) for categorical variables. A confidence interval calculation for binomial proportions was generated for the study's primary endpoint (functionally favorable survival to hospital discharge) and secondary endpoint (functionally favorable 3-month survival) using the Wilson-Score method.

\section{Role of the funding source}

The funding source, the Helmsley Charitable Trust, had no role in the study design, collection, analysis, or interpretation of data, the writing or editing of the manuscript, or the decision to submit the work for publication.

\section{Results}

\subsection{Enrollment and characteristics}

From December 1, 2019 to April 1, 2020, there were 151 patients with VF/VT OHCA. Of these, 97/151 (64\%) were refractory VF/VT OHCA. Of these 97 refractory VF/VT OHCA patients, 60 , plus 3 primary pulseless electrical activity (PEA) patients, were transported to the 3 ECMO Initiation Hospitals. Of these transported patients, 5/63 (8\%) did not meet MMRC criteria, resulting in 92\% (58/63) accuracy of patient selection for transport and $100 \%$ capture of all 58 refractory VF/VT OHCA patients meeting MMRC criteria during the study period. Of the 58 meeting MMRC criteria (14.5 patients/month), 13/58 (22\%) had $\geq 2$ resuscitation discontinuation criteria on arrival and were pronounced dead, 45/58 (78\%) received full resuscitation efforts (ECMO cannulation and CCL angiography/PCI), 4/58 (7\%) were declared dead prior to transfer to the ECMO ICU due to refractory shock or failure to achieve an organized rhythm in the CCL, and 41/ $58(71 \%)$ were transferred to the ECMO ICU (Fig. 3). The day of the week and time of day these patients were treated is shown in Fig. $4 \mathrm{~A}$ and $4 \mathrm{~B}$.

Of the patients meeting MMRC criteria, mean age was $57 \pm 14$ years; $8 / 58$ (14\%) patients were $<40$ years old, $23 / 58$ (40\%) were $40-60$ years old, and $27 / 58$ (47\%) were $61-75$ years old; $46 / 58$ (79\%) were male; 49/58 (84\%) were white, 4/58 (7\%) were black, and $5 / 58(9 \%)$ other. Known comorbidities included coronary artery disease (18/58 [31\%]), diabetes mellitus (14/58 [24\%]), hypertension (24/58 [41\%]), hyperlipidemia (19/58 [33\%]), smoking $(21 / 58[36 \%])$, alcoholism $(7 / 58[12 \%])$ and congestive heart failure $(6 / 58[10 \%])$.

\section{Program performance and benchmarks}

\subsection{EMS agencies}

Accuracy of EMS agency patient selection was 58/63 (92\%). Despite a benchmark of $<15 \mathrm{~min}$, paramedics were on-scene a mean of $22.0 \pm 8.9$ min prior to transport; > $15 \mathrm{~min}$ in $66 \%$ of cases. Mean time from 911 call to patient arrival at the 3 ECMO Initiation Hospitals was $46.9 \pm 12.3 \mathrm{~min}$. Cardiac arrest characteristics and EMS treatments are shown in Table 2.

\subsection{ECMO initiation hospitals}

The number and proportion of eligible cardiac arrest patients received at the three ECMO Initiation Hospitals were 19/58 (33\%), 25/ 58 (43\%), and $14 / 58(24 \%)$.

\subsection{Discontinuation criteria on ED arrival}

Prolonged resuscitation resulted in severe metabolic derangement for many patients with a mean lactic acid of $12.5 \pm 4.2 \mathrm{mmol} / \mathrm{L}$, $\mathrm{pH}$ of $6.98 \pm 0.02$, and $\mathrm{PaO} 2$ of $87 \pm 109$. Thirteen of 58 patients (22\%) met resuscitation discontinuation criteria and were declared dead prior to any attempted ECMO cannulation because of meeting both lactic acid and $\mathrm{PaO} 2$ criteria. 


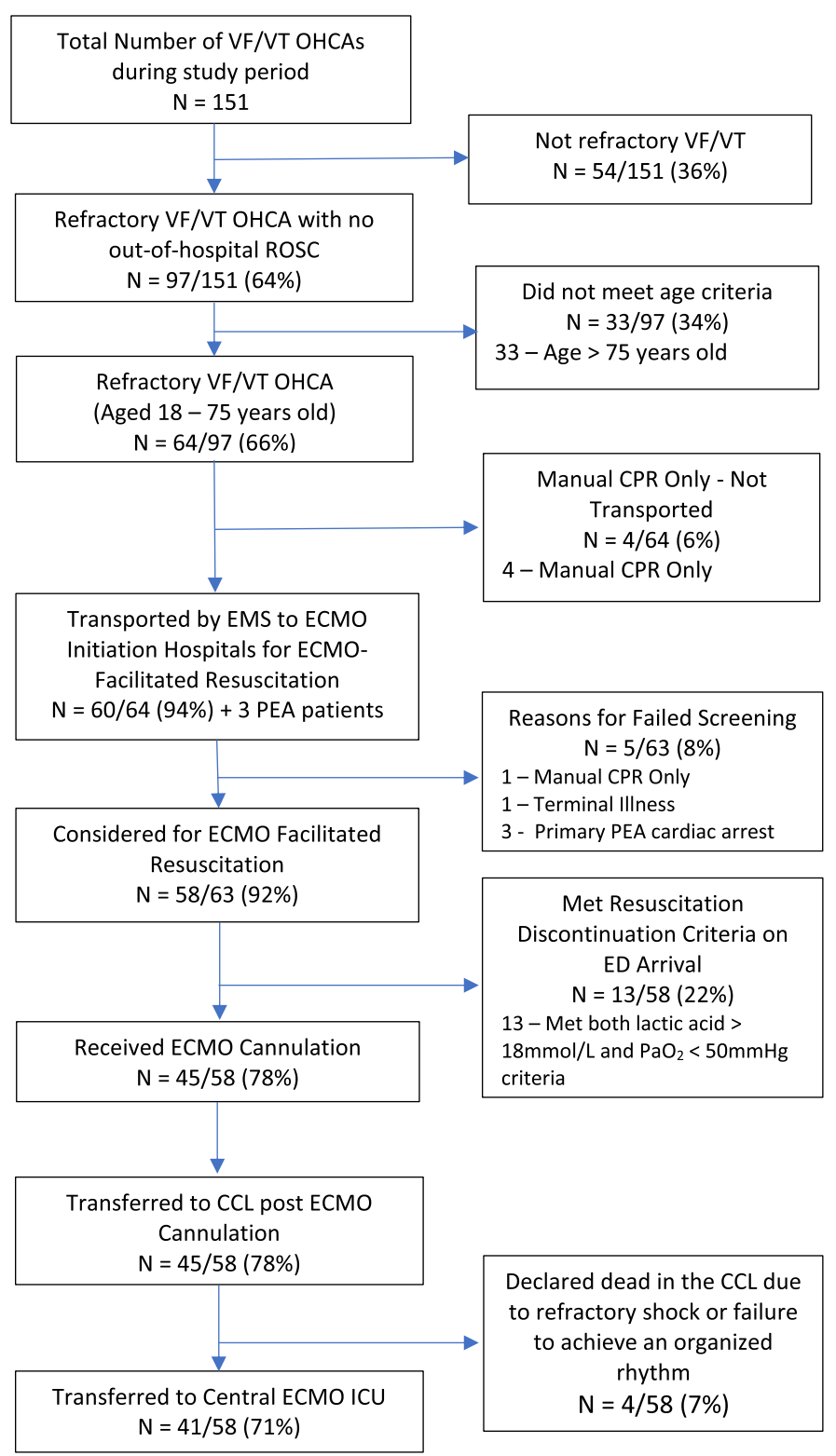

Fig. 3. Patient flow diagram for patients treated by the MMRC Program.

\subsection{Mobile ECMO cannulation team}

The mobile ECMO cannulation team made 63/63 (100\%) safe, rapid responses to the 3 ECMO Initiation Hospitals; mean response time was $14.9 \pm 5.7 \mathrm{~min}$ (benchmark $<15 \mathrm{~min}$ ); $71 \%$ of cases had response times $<15 \mathrm{~min}$. Mean time from ECMO-eligible patient arrival to ECMO initiation was $14.4 \pm 6.1 \mathrm{~min}$; $63 \%$ received ECMO in $<15 \mathrm{~min}$. All ECMO-eligible cannulations were successful without complications (45/45 [100\%]). Mean duration of professional CPR was $52.2 \pm 17.0 \mathrm{~min}$ (benchmark < $60 \mathrm{~min}$ ).

\section{5. $C C L$}

Patients were taken to the CCL at the ECMO Initiation Hospital within 2 h of 911 call in 21/45 (47\%) of cases; mean time was $121 \pm 56 \mathrm{~min}$. Coronary angiography was performed in 45/58 (78\%) patients; $29 / 45$ (64\%) had severe coronary artery disease and 22/29 $(85 \%)$ received $\mathrm{PCI}$. There were $1.8 \pm 1.2$ stents placed in all vessels/ patient. Culprit vessels are shown in Table 2.
10.6. Transport to the central ecmo icu

Transport to the central ECMO ICU occurred without adverse events in all cases (41/41 [100\%]).

\subsection{Central ECMO ICU care}

All patients received therapeutic hypothermia (goal temperature of $34{ }^{\circ} \mathrm{C}$ ). For survivors, cardiac function recovered to a mean left ventricular ejection fraction of $51 \pm 14 \%$ at hospital discharge. Of the 25 functionally favorable survivors, intubation continued a mean of $10.2 \pm 7.5$ days; hospitalization lasted $18.9 \pm 8.6$ days; $80 \%$ were discharged to an acute rehabilitation facility prior to discharge home. Patients who died were hospitalized a mean of $7.0 \pm 13$ days. Cause of death in non-survivors was as follows: 13 met discontinuation criteria on arrival at the ECMO Initiation Hospital, 4 died in the CCL due to refractory shock or failure to achieve an organized rhythm despite ECMO, 7 had acute brain death, 7 had severe anoxic brain injury. We did not observe any severe ECMO-machine related 
A

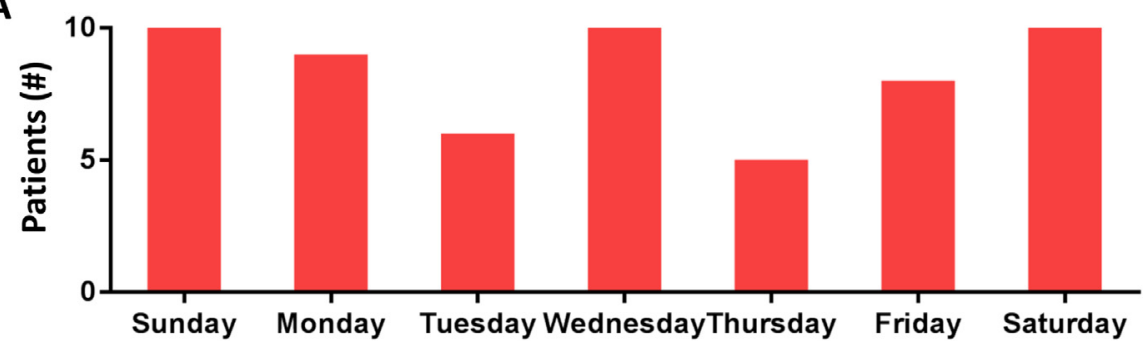

B

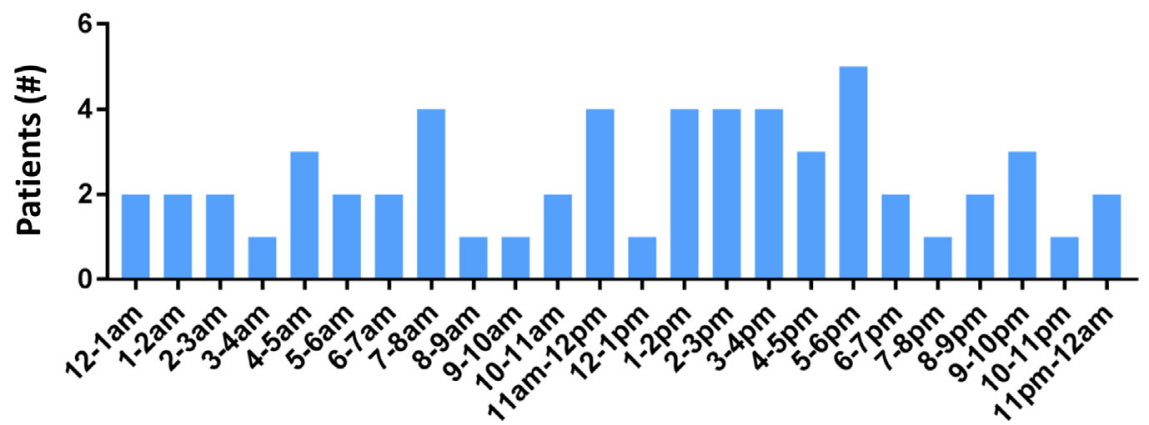

Fig. 4. Timing of ECMO-facilitated Resuscitation Cases. A. Frequency of cases by day of the week. B. Frequency of cases by time of day.

complications including thrombosis, air-embolism, or electronics failure. Four of 45 (9\%) patients had access site bleeding requiring > 3 units packed red blood cells. A single, unexplained increase in circuit transmembrane resistance led to a mandatory circuit exchange 15 min after ECMO initiation.

\subsection{Survival}

Survival to hospital discharge occurred in 27/58 (47\%) and 25/58 (43\% [CI: $31-56 \%$ ]) had functionally favorable survival (CPC of 1 or 2). Of those discharged from the hospital, $100 \%$ were alive at 3 months

Table 2

Results of MMRC process characteristics, performance metrics, and benchmarks.

\begin{tabular}{|c|c|}
\hline Characteristic/Performance Metric/Benchmark & Value \\
\hline \multicolumn{2}{|l|}{10 EMS Agencies } \\
\hline Accuracy of ECMO-facilitated Resuscitation Patient Selection [N, (\%)] & $58 / 63(92 \%)$ \\
\hline 911 to first responder arrival (minutes) & $7.2 \pm 3.6$ \\
\hline EMS On-Scene time (minutes; benchmark < 15 min) & $22.0 \pm 8.9$ \\
\hline 911 to patient arrival at $\mathrm{ED}$ (minutes) & $46.9 \pm 12.3$ \\
\hline Public Location [N, (\%)] & $18 / 58(31 \%)$ \\
\hline Bystander witnessed $[\mathrm{N},(\%)]$ & $42 / 58(72 \%)$ \\
\hline Bystander /Dispatched Assisted CPR [N, (\%)] & $32 / 58(55 \%)$ \\
\hline \multicolumn{2}{|l|}{ Out-of-Hospital Airway [N, (\%)] } \\
\hline Bag-valve-mask only & $6 / 58(10 \%)$ \\
\hline Supraglottic Airway & $22 / 58(38 \%)$ \\
\hline Endotracheal Intubation & $30 / 58(52 \%)$ \\
\hline Epinephrine doses (1 mg) & $3.4 \pm 0.7$ \\
\hline Amiodarone (mg dose) & $387 \pm 75$ \\
\hline Number of shocks by EMS & $5.3 \pm 2.1$ \\
\hline Intermittent ROSC prior to ED arrival [N, (\%)] & $16 / 58(28 \%)$ \\
\hline \multicolumn{2}{|l|}{ ECMO Initiation Hospitals } \\
\hline $1 \mathrm{~N}(\%)$ & $19 / 58(33 \%)$ \\
\hline $2 \mathrm{~N}(\%)$ & $25 / 58(43 \%)$ \\
\hline $3 \mathrm{~N}(\%)$ & $14 / 58(24 \%)$ \\
\hline \multicolumn{2}{|l|}{ Perfusion Discontinuation Criteria on ECMO Initiation Hospital Arrival } \\
\hline Initial lactic Acid (mmol/L) & $12.5 \pm 4.2$ \\
\hline Initial $\mathrm{pH}$ & $6.98 \pm 0.21$ \\
\hline Initial arterial oxygen, $\mathrm{PaO} 2,(\mathrm{~mm} \mathrm{Hg})$ & $87 \pm 109$ \\
\hline Initial serum bicarbonate, $\mathrm{mg} / \mathrm{dL}$ & $16.3 \pm 5.6$ \\
\hline End Tidal CO2 (\%) & $35 \pm 16$ \\
\hline Proportion of patients meeting discontinuation criteria $[\mathrm{N},(\%)]$ & $13 / 58(22 \%)$ \\
\hline \multicolumn{2}{|l|}{ Mobile ECMO Cannulation Team } \\
\hline Response time to $\mathrm{ED}$ (mean, minutes; benchmark < 15 min) & $14.9 \pm 5.7$ \\
\hline Number of Safe Responses [N, (\%)] & $63 / 63(100 \%)$ \\
\hline Patient ED Arrival to ECMO Cannulation (mean, minutes; benchmark $<15$ min) & $14.4 \pm 6.1$ \\
\hline Duration of Patient Professional CPR (minutes; benchmark < 60 min) & $52.2 \pm 17.0$ \\
\hline Cannulation success rate $[\mathrm{N},(\%)]$ & $45 / 45(100 \%)$ \\
\hline
\end{tabular}


Table 2 (Continued)

\begin{tabular}{|c|c|}
\hline Characteristic/Performance Metric/Benchmark & Value \\
\hline Cannulation complication rate $[\mathrm{N},(\%)]$ & $0 / 45(0 \%)$ \\
\hline \multicolumn{2}{|l|}{ Cardiac Catheterization Laboratory (CCL) } \\
\hline 911 to Cardiac Catheterization Lab Time (mean; min; benchmark < 120 min) & $121 \pm 56$ \\
\hline Angiography performed $[\mathrm{N},(\%)]$ & $45 / 58(78 \%)$ \\
\hline Presence of severe coronary artery disease $[\mathrm{N},(\%)]$ & $29 / 45(64 \%)$ \\
\hline Percutaneous coronary intervention performed $[\mathrm{N},(\%)]$ & $22 / 29(85 \%)$ \\
\hline \multicolumn{2}{|l|}{ Culprit vessel [Number, (\%) of Patients] } \\
\hline Left main coronary artery & $2 / 45(4 \%)$ \\
\hline Left anterior descending & $12 / 45(27 \%)$ \\
\hline Left circumflex & $0 / 45(0 \%)$ \\
\hline Right coronary artery & $8 / 45(18 \%)$ \\
\hline Chronic total occlusions & $10 / 45(22 \%)$ \\
\hline Total stents placed in all vessels (mean) & $1.8 \pm 1.2$ \\
\hline \multicolumn{2}{|l|}{ Transport to ECMO Intensive Care Unit } \\
\hline Number of transports without adverse events [N, (\%)] & $41 / 41(100 \%)$ \\
\hline \multicolumn{2}{|l|}{ Centralized ECMO Intensive Care Unit } \\
\hline Therapeutic Hypothermia Provided ( $24 \mathrm{~h}$; goal temperature $\left.34^{\circ} \mathrm{C}\right)[\mathrm{N},(\%)]$ & $41 / 41(100 \%)$ \\
\hline 24-hour LVEF on echocardiogram in ICU (\%) & $13 \pm 13$ \\
\hline LVEF on hospital discharge (\%) & $51 \pm 14$ \\
\hline Tracheostomy [N, (\%)] & $5 / 41(12 \%)$ \\
\hline Percutaneous endoscopic gastrostomy tube $[\mathrm{N},(\%)]$ & $5 / 41(12 \%)$ \\
\hline \multicolumn{2}{|l|}{ ECMO-Related Complications } \\
\hline Circuit thrombosis [N, (\%)] & $0 / 45(0 \%)$ \\
\hline Air-embolism [N, (\%)] & $0 / 45(0 \%)$ \\
\hline Access site bleeding requiring $>3$ units PRBCs $[\mathrm{N},(\%)]$ & $4 / 45(9 \%)$ \\
\hline Ischemic Limb requiring intervention (fasciotomy, amputation) [N, (\%)] & $0 / 45(0 \%)$ \\
\hline Circuit failure (increased transmembrane resistance) $[\mathrm{N},(\%)]$ & $1 / 45(2 \%)$ \\
\hline \multicolumn{2}{|l|}{ Time to Decannulation, days } \\
\hline Survivors & $4.2 \pm 1.5$ \\
\hline Non-survivors & - \\
\hline \multicolumn{2}{|l|}{ Time to Extubation, days } \\
\hline Survivors & $10.2 \pm 7.5$ \\
\hline Non-survivors & - \\
\hline \multicolumn{2}{|l|}{ Length of ICU Stay, days } \\
\hline Survivors & $15.1 \pm 8.1$ \\
\hline Non-survivors & $7.0 \pm 13$ \\
\hline \multicolumn{2}{|l|}{ Hospital Length of Stay, days } \\
\hline Survivors & $18.9 \pm 8.6$ \\
\hline Non-survivors & $7.0 \pm 13$ \\
\hline \multicolumn{2}{|l|}{ Outcomes } \\
\hline Survival to hospital discharge $[\mathrm{N},(\%)]$ & $27 / 58(47 \%)$ \\
\hline Functionally favorable survival to hospital discharge (CPC 1 or 2$)[\mathrm{N},(\%), \mathrm{CI}]$ & $25 / 58$ (43\% [CI: $31-56 \%])$ \\
\hline Survival to 3 months & $25 / 58(43 \%)$ \\
\hline Functionally favorable 3-month survival (CPC 1 or 2 ) [N, (\%), CI] & $25 / 58$ (43\% [CI: $31-56 \%])$ \\
\hline HOSPITAL DISCHARGE Cerebral Performance Category (mean; $N=27$ ) & $1.6 \pm 0.7$ \\
\hline $\mathrm{CPC} 1$ & $12 / 58(21 \%)$ \\
\hline $\mathrm{CPC} 2$ & $13 / 58(22 \%)$ \\
\hline $\mathrm{CPC} 3$ & $0 / 58(0 \%)$ \\
\hline $\mathrm{CPC} 4$ & $2 / 58(3 \%)$ \\
\hline CPC 5 & $31 / 58(54 \%)$ \\
\hline 3-MONTH Cerebral Performance Category (mean; $N=27$ ) & $1.3 \pm 0.7$ \\
\hline $\mathrm{CPC} 1$ & $19 / 58(33 \%)$ \\
\hline $\mathrm{CPC} 2$ & $6 / 58(10 \%)$ \\
\hline $\mathrm{CPC} 3$ & $0 / 58(0 \%)$ \\
\hline $\mathrm{CPC} 4$ & $2 / 58(3 \%)$ \\
\hline CPC 5 & $31 / 58(54 \%)$ \\
\hline
\end{tabular}

Table 2: MMRC Process Characteristics, Performance Metrics, and Benchmarks. Values are $\mathrm{n}(\%)$ or mean \pm standard deviation. MMRC = Minnesota Mobile Resuscitation Consortium EMS = Emergency Medical Service, $\mathrm{ECMO}=$ Extracorporeal Membrane Oxygenation, $\mathrm{ED}=$ Emergency Department; mmol = millimoles; $L=$ liter; $\mathrm{pH}=$ potential of hydrogen; $\mathrm{PaO} 2$ = partial pressure of oxygen in arterial blood; $\mathrm{mmHg}=$ millimeters of mercury; O2Sat = oxygen saturation; ETCO2 = end-tidal carbon dioxide; EIH = ECMO Initiation Hospital; CPR= cardiopulmonary resuscitation; $\mathrm{CCL}=$ Cardiac Catheterization Laboratory, $\mathrm{PRBCs}=$ packed red blood cells; $\mathrm{CPC}=$ Cerebral Performance Category; $\mathrm{CI}=$ Confidence Interval.

with CPC 1 or $2(25 / 58,43 \%$ [CI: $31-56 \%])$. Mean CPC score was $1.6 \pm 0.7$ and $1.3 \pm 0.7$, at hospital discharge and 3-months, respectively. The CPC score of all patients at hospital discharge and 3 months is shown in Table 2.

\section{Discussion}

Refractory VF/VT OHCA is most often associated with severe coronary artery disease and acute occlusion with minimal survival unless treatment is provided to reverse the underlying, presumably causative, pathophysiology [9]. ECMO-facilitated resuscitation acts acutely as a bridge, making emergent angiography and PCI feasible. Subsequently, it facilitates hemodynamic stability, supporting ICU treatment of multi-organ injury and recovery of stunned myocardium.

Each component of care is crucial and significantly contributes to survival, similar to the American Heart Association's links in the Chain of Survival [16-18]. It is not the ECMO technology that makes the difference. It is the ECMO-facilitated resuscitation program that has potential to transform outcomes for this patient population. The 
MMRC program described, and the $43 \%$ functionally favorable survival observed, provides a potential model of this approach for other communities.

A number of studies afford historical perspective and comparison with the $43 \%$ functionally favorable survival rate seen in this study $[5,6,8,13]$. The MRC previously collected data from the Minneapolis/ St. Paul environs from January 1, 2013 to December 31, 2014 in patients with VF/VT OHCA aged 18-75 who did not have ROSC before intravenous/intraosseous amiodarone and received standard ACLS treatment. Survival to hospital discharge with CPC 1 or 2 was $14 / 170$ (8.2\%) [5]. A prior MRC study used the Amiodarone, Lidocaine, or Placebo in Out-of-Hospital Cardiac Arrest (ALPS) Study, [6] a randomized, multi-center clinical trial, as a historical control, identifying adults aged 18-75 with VF/VT OHCA enrolled in the amiodarone arm with VF/VT refractory to at least 1 shock. Survival to hospital discharge with CPC 1 or 2 was 148/654 (23\%) [8]. These historical comparisons are limited and unmatched for other factors known to affect outcome from cardiac arrest. Perhaps, the most valid available comparison is the control group, receiving standard ACLS treatment, in the first prospective, randomized clinical trial of ECMO-facilitated resuscitation versus standard ACLS treatment for refractory VF/VT OHCA, the ARREST trial [13]. The study was performed with complete independence and without patient overlap to this study, implemented in the Minneapolis/St. Paul environs by the same group of core investigators, and met all inclusion criteria for the MMRC program. Survival to hospital discharge was $1 / 15$ (6.7\%), survival to hospital discharge with CPC 1 or 2 was $0 / 15(0 \%)$ and 3- and 6-month survival was $0 / 15(0 \%)$ [13].

Fundamental to the success of the program was the recognition that low patient volumes would not support provision of this complex and costly therapy. Multiple program components were essential for success including: 1) structuring the program as a nonprofit entity (establishing its neutrality), 2) involvement of participating healthcare systems by representation on the board of directors (engendering process investment and support) and selection of ECMO cannulation team members from each system, 3) selecting a modest number of well-trained mobile ECMO cannulation team members (assuring high quality performance, high patient volume, and skill maintenance), 4) selection of ECMO Initiation Hospitals and cost sharing of services with the healthcare systems (providing economic incentive, program investment, and expanded communitywide footprint), 5) integrated and dedicated EMS medical directors and providers (providing accurate, high quality care, and continuous feedback), 6) a single, centralized ECMO ICU (favorable economics, 24/7 emergent availability of all necessary consulting surgical and medical specialties, high patient volume, and continuity of care), and 7) establishing community-wide data collection capability (monitoring performance, benchmarking, and continuous quality improvement). These core components provided the patient volume necessary for optimal care, good functionally favorable survival rates justifying program continuation, economy of scale, lower costs, and sustainability.

The MMRC felt safety of the program was established with 63/63 (100\%) safe ECMO cannulation team responses and $41 / 41(100 \%)$ transfers to the ECMO ICU without adverse events. Forty-five of 45 (100\%) cannulations occurred without complication.

There were multiple challenges. A lack of community experience treating this patient population necessitated rigorous education/ training programs, particularly EMS providers and ECMO cannulation team members. Capability to monitor performance required centralized data collection from diverse stakeholders including EMS, ECMO ICU, and multiple healthcare systems.

Some benchmarks and goals were not achieved. The mean duration of professional CPR only decreased 5-8 min compared to prior cohort studies from a single center [5,7-9]. Paramedic scene time was longer than $15 \mathrm{~min}$ for diverse reasons including patient extraction delays. Reducing CPR time is a high priority as it remains a critical determinant of survival [8].

The time from arrest to coronary angiography was also longer than benchmarked. The goal of two hours was established because coronary occlusion is a common cause of refractory VF/VT OHCA [9]. Reducing time to CCL remains a priority, although timing of this component's relative contribution to survival is unknown.

This study has limitations. There may be unique aspects which promoted development of this specific, successful program. Other locations may require customization to develop similar programs. Nonetheless, we recommend inclusion of the essential components and principles discussed, whenever possible. Quality improvement and the MMRC program development is still ongoing. Aspects may evolve over time. The moderate size of this patient cohort makes meaningful subgroup comparisons insensitive to differences. Assessment of larger cohorts may identify differences between subpopulations of interest. A detailed cost analysis was beyond the scope of this study and remains to be determined.

In summary, this first, community-wide ECMO-facilitated resuscitation program in the US demonstrated $100 \%$ successful cannulation, 43\% functionally favorable survival rates at hospital discharge and 3 months, as well as safety. The program provides a potential model of this approach for other communities.

\section{Author contributions}

All authors made substantial contributions to conception and design of the study. JAB, KH, LH, MK, TPA, and DY performed data acquisition, analysis, and interpretation. JB, TPA, and DY drafted the manuscript. All authors participated in critical revision of the manuscript and approval of the final submitted version. All authors accept responsibility for the integrity of the data analyzed.

\section{Declaration of Competing Interest}

All authors report having received funding from the Leona $M$. and Harry B. Helmsley Charitable Trust. JAB, TPA, and DY also received funding from the National Heart, Lung, and Blood Institute.

\section{Acknowledgments}

The authors would like to acknowledge the efforts of the Minneapolis and St. Paul metropolitan community, first responders, and emergency medical services providers who work every day to save lives in our community. We also appreciate the tireless work of the nurses, physicians, and allied hospital staff who provide the postarrest care for these critically ill patients. The results described here would not be possible without strength in every link in the chain of survival.

\section{Data sharing statement}

The data reported in this study are available from the Minnesota Mobile Resuscitation Consortium upon reasonable request.

\section{Funding}

Project funding was received from the Helmsley Charitable Trust. $J A B, T P A$, and DY also received funding from the National Heart, Lung, and Blood Institute.

\section{References}

[1] Becker LB, Aufderheide TP, Graham R. Strategies to improve survival from cardiac arrest: a report from the institute of medicine. JAMA 2015;314(3):223-4. 
[2] Benjamin EJ, Muntner P, Alonso A, et al. Heart disease and stroke statistics-2019 update: a report from the American heart association. Circulation 2019;139(10): e56-e528.

[3] Zive DM, Schmicker R, Daya M, et al. Survival and variability over time from out of hospital cardiac arrest across large geographically diverse communities participating in the resuscitation outcomes consortium. Resuscitation 2018;131:74-82.

[4] Yannopoulos D, Bartos JA, Aufderheide TP, et al. The evolving role of the cardiac catheterization laboratory in the management of patients with out-of-hospital cardiac arrest: a scientific statement from the American heart association. Circulation 2019;139(12):e530-e52.

[5] Yannopoulos D, Bartos JA, Martin C, et al. Minnesota resuscitation consortium's advanced perfusion and reperfusion cardiac life support strategy for out-of-hospital refractory ventricular fibrillation. J Am Heart Assoc 2016;5(6):e003732.

[6] Kudenchuk PJ, Brown SP, Daya M, et al. Amiodarone, lidocaine, or placebo in outof-hospital cardiac arrest. N Engl J Med 2016;374(18):1711-22.

[7] Bartos JA, Carlson K, Carlson C, et al. Surviving refractory out-of-hospital ventricular fibrillation cardiac arrest: critical care and extracorporeal membrane oxygenation management. Resuscitation 2018;132:47-55.

[8] Bartos JA, Grunau B, Carlson C, et al. Improved survival with extracorporeal cardiopulmonary resuscitation despite progressive metabolic derangement associated with prolonged resuscitation. Circulation 2020;141(11):877-86.

[9] Yannopoulos D, Bartos JA, Raveendran G, et al. Coronary artery disease in patients with out-of-hospital refractory ventricular fibrillation cardiac arrest. J Am Coll Cardiol 2017;70(9):1109-17.

[10] Lamhaut L, Hutin A, Puymirat E, et al. A pre-hospital extracorporeal cardio pulmonary resuscitation (ECPR) strategy for treatment of refractory out hospital cardiac arrest: an observational study and propensity analysis. Resuscitation 2017;117:109-17.

[11] Stub D, Bernard S, Pellegrino V, et al. Refractory cardiac arrest treated with mechanical CPR, hypothermia, ECMO and early reperfusion (the CHEER trial). Resuscitation 2015;86:88-94.
[12] Kagawa E, Dote K, Kato M, et al. Should we emergently revascularize occluded coronaries for cardiac arrest?: rapid-response extracorporeal membrane oxygenation and intra-arrest percutaneous coronary intervention. Circulation 2012;126 (13):1605-13.

[13] Yannopoulos D, Bartos JA, Raveendran G, Walser E, Connett J, Murray TA, Collins G, Zhang L, Kalra R, Kosmopoulos M, John R, Shaffer A, Frascone RJ, Wesley K, Conterato M, Biros M, Aufderheide TP. Advanced reperfusion strategies for refractory ventricular fibrillation out-of-hospital cardiac arrest: the ARREST trial. Lancet 2020.

[14] Nasr VG, Raman L, Barbaro RP, Guner Y, Tonna J, Ramanathan K, Pappalardo F, Thiagarajan RR, Alexander PMA. ELSO registry scientific oversight committee. highlights from the extracorporeal life support organization registry: 2006-2017. ASAIO J 2019 Aug;65(6):537-44.

[15] Elm EV, Altman DG, Egger M, Pocock SJ, Gøtzsche PC, Vandenbroucke JP, STROBE Initiative. The strengthening the reporting of observational studies in epidemiology (STROBE) statement: guidelines for reporting observational studies. Lancet 2007:370(9596):1453-7.

[16] Travers AH, Rea TD, Bobrow BJ, et al. Part 4: CPR overview: 2010 American heart association guidelines for cardiopulmonary resuscitation and emergency cardiovascular care. Circulation 2010;122(18 Suppl 3):S676-84

[17] Kronick SL, Kurz MC, Lin S, et al. Part 4: systems of care and continuous quality improvement: 2015 American heart association guidelines update for cardiopulmonary resuscitation and emergency cardiovascular care. Circulation 2015;132 (18 Suppl 2):S397-413.

[18] Cummins RO, Ornato JP, Thies WH, Pepe PE. Improving survival from sudden cardiac arrest: the "chain of survival" concept. A statement for health professionals from the advanced cardiac life support subcommittee and the emergency cardiac care committee, American heart association. Circulation 1991;83(5):1832-47. 